\title{
Minimally Invasive Total Hip Arthroplasty: A Comparison of Restoring Hip Biomechanics With and Without a Traction Table
}

\author{
FLORIAN LENZE $^{1}$, FLORIAN HINTERWIMMER ${ }^{1}$, LISA FLECKENSTEIN $^{1}$, \\ IGOR LAZIC $^{1}$, DIETMAR DAMMERER ${ }^{2}$, RÜDIGER VON EISENHART-ROTHE ${ }^{1}$, \\ NORBERT HARRASSER ${ }^{1}$ and FLORIAN POHLIG ${ }^{1}$ \\ ${ }^{1}$ Department of Orthopedics and Sports Orthopedics, Klinikum rechts der Isar, \\ Technical University Munich, Munich, Germany; \\ ${ }^{2}$ Department of Orthopedics and Traumatology, Medical University of Innsbruck, Innsbruck, Austria
}

\begin{abstract}
Background/Aim: The aim of the study was to analyze operative time and restoration of hip biomechanics in total hip arthroplasty (THA) via direct anterior approach (DAA) with and without the use of a traction table. Patients and Methods: We retrospectively compared 97 cases where a traction table was used to 92 cases without a table. Ninetyseven patients received THA with a traction table $\left(A M I S^{(B)}\right.$ technique) and 92 patients with conventional DAA. Postoperative standard radiographs were used to analyze offset parameters and leg length. Furthermore, time for patient positioning and cut-to-suture time were evaluated. Results: Cut-to-suture time was statistically significantly shorter in the traction table group $(p=0.001)$, whereas analysis of offset parameters (acetabular, femoral and combined) was comparable between the two groups ( $p=0.31, p=0.95, p=0.42$ ). Postoperative leg length was statistically significantly different with and without traction table use ( $p=0.02)$. Conclusion: Both methods enable restoration of hip biomechanics with high accuracy. Further studies with prospective study designs and larger sample sizes may be needed to confirm these results.
\end{abstract}

Total hip arthroplasty (THA) is one of the most successful procedures in orthopedic surgery (1). Due to demographic development, THA is projected to grow more than $70 \%$ by

This article is freely accessible online.

Correspondence to: Florian Lenze, MD, Department of Orthopedics and Sports Orthopedics, Klinikum rechts der Isar, Technical University Munich, Ismaninger Str. 22, 81675 Munich, Germany. Tel: +498941402271, e-mail: Florian.Lenze@mri.tum.de

Key Words: Minimally invasive total hip arthroplasty, traction table, hip biomechanics, biomechanical restoration, direct anterior approach.
2030 in the U.S. (2). In the past, research in THA was mainly focused on implant designs, whereas today the optimization of the surgical procedure has gained significant importance. In this context, minimally invasive surgical approaches, which are defined by an incision length of $10-12 \mathrm{~cm}$, became increasingly popular $(3,4)$. According to various authors, minimally invasive THA is associated with less blood loss, lower perioperative pain levels, lower dislocation rates and earlier return to daily life activities (5-7). On the contrary, other studies found no benefit of minimal incision THA over standard approaches $(5,8,9)$.

One of the most popular minimally invasive hip approaches is the direct anterior approach (DAA) due to its tissue sparing technique using an intermuscular and internervous path between $\mathrm{M}$. tensor fascia latae and $\mathrm{M}$. rectus femoris (10). Despite the growing popularity of DAA, concerns remain regarding implant positioning due to a flat learning curve and the limited exposure $(11,12)$.

THA via DAA can be performed on a standard operation table or with a traction table. The use of a traction table may enhance surgical exposure, while patient positioning is more time-consuming and intraoperative assessment of leg length is limited (10). However, to date, it remains unclear if the use of a traction table facilitates shorter surgery duration and optimized restoration of hip biomechanics due to the improved exposure compared to the conventional DAA technique (13).

For the present study we hypothesized that: 1) surgical duration is shorter when DAA THA is performed with a traction table and 2) restoration of hip biomechanics is similar in both techniques.

\section{Patients and Methods}

The study was approved by the local ethics committee (project number 148/15). We retrospectively enrolled all patients who were treated with cementless THA via DAA between 2013 and 2015 at an Orthopaedic University clinic. 


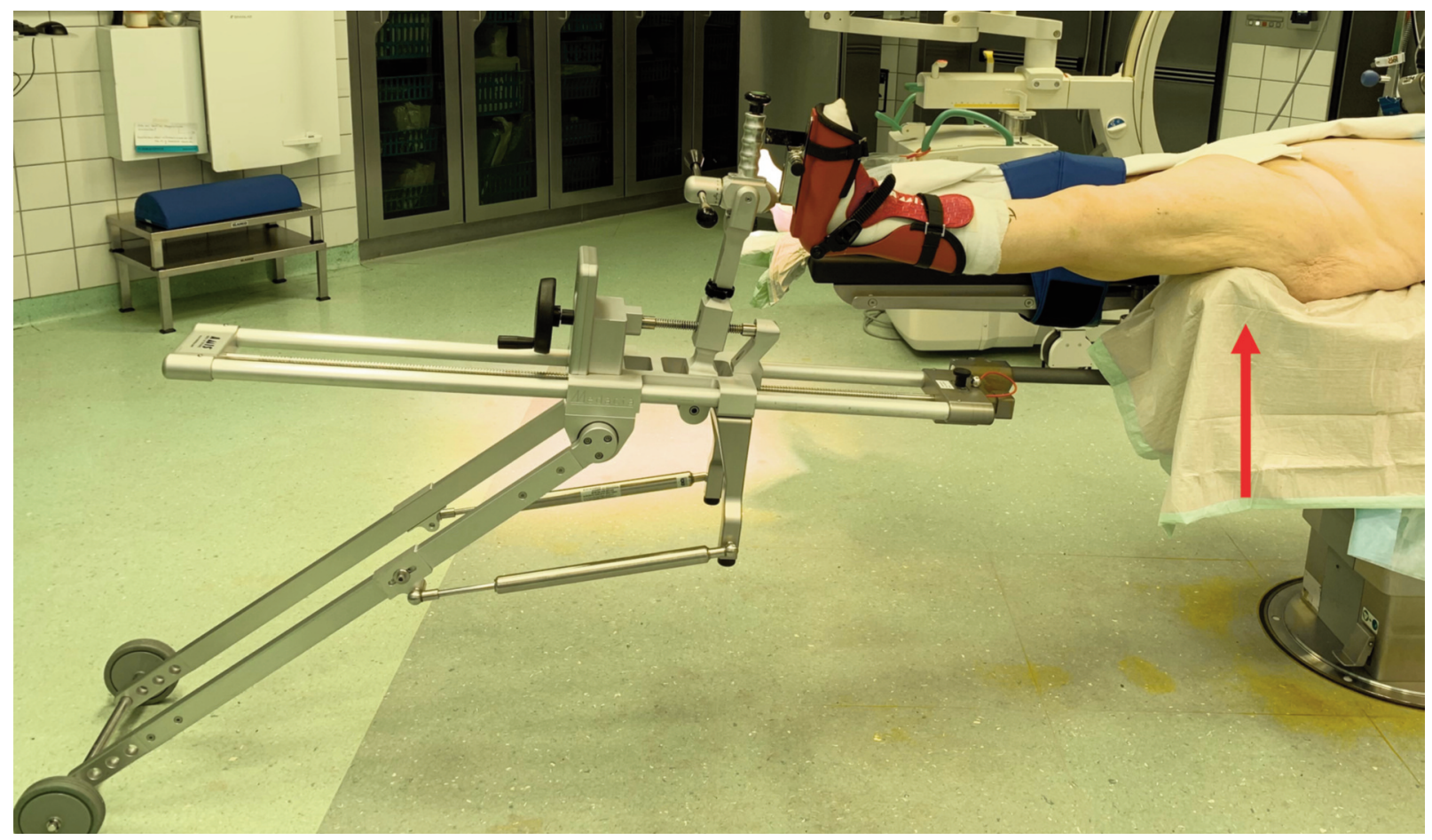

Figure 1. AMIS mobile leg positioner. A hypomochlion (red arrow) lifts the coxal end of the femur when the leg is moved into hyperextension.

Inclusion criteria were diagnosis of osteoarthritis of the hip joint and age of 18 years and above. Exclusion criteria were cemented fixation of the prosthesis, pre-existing severe deformities, BMI > 35 $\mathrm{kg} / \mathrm{m}^{2}$, severe comorbidities [ $>$ ASA (American Society of Anesthesiologists) III] and previous bone surgeries on the index leg.

Surgical procedure. All hip replacement surgeries were performed via DAA either with a conventional operation table or a traction table. Four senior surgeons performed the hip joint replacements.

Patients received either an AMIStem ${ }^{\circledR}$ (Medacta International, Castel San Pietro, Switzerland) or a Fitmore ${ }^{\circledR}$ stem (Zimmer Biomet, Warsaw, IN, USA). In all patients, either an Allofit Alloclassic ${ }^{\circledR} \operatorname{cup}\left(\right.$ Zimmer Biomet) or a Versafit ${ }^{\circledR}$ cup (Medacta International) were used.

For conventional DAA THA a standard operation table with the patient in supine position was used. The pelvis was located over the table break to allow hyperextension for stem preparation.

All other DAA THAs were performed with the use of the AMIS ${ }^{\circledR}$ Mobile Leg Positioner (Medacta International) (Figure 1). Exposure was facilitated by traction, external rotation, and hyperextension.

Measurements. All surgical procedures were preoperatively planed with a digital-planning software using standard anterior-posterior low pelvic radiographs with a $25 \mathrm{~mm}$ reference ball (mediCAD; Hectec GmbH, Altdorf, Germany). A recent published study on preoperative $2 \mathrm{D}$ templating revealed a high accuracy when using the mediCAD program (14). Implant size and position was determined, femoral as well as acetabular offset and leg length discrepancy were taken into account. Restoration of offset was defined to be equal to the contralateral hip joint. The target for leg length reconstruction was for the operated leg to be the same length as the contralateral leg. For estimation of lower limb length discrepancy, a line tangential and parallel to the most inferior point of the ischia was constructed (15). A second line was then placed at the most medial aspect of the lesser trochanter and then the distance between the two lines was measured on both sides.

Femoral offset was defined as the distance from the center of rotation of the femoral head to the line of the femoral metaphysis canal $(16,17)$. The acetabular offset was defined as the perpendicular distance from the center of rotation of the femoral head to the vertical trans-teardrop line (17). Acetabular offset and femoral offset were then summarized as combined offset. An increase in offset assigned positive values and a decrease assigned negative values.

Postoperative radiographs were routinely performed 3 days after surgery. Offset and leg length discrepancy were then analyzed using the same digital planning software. Three blinded observers performed the radiographic assessment by using a standardized protocol.

Time for patient positioning and cut-to-suture time were extracted from the perioperative documentation. Time for patient positioning was measured from the end of anesthesia preparation to final positioning of patients for surgery. Cut-to-suture time was measured from incision to suture.

Statistical analysis. Statistical analyses were performed using SPSS 21.0 software (IBM SPSS, Armonk, NY, USA). Normal distribution was tested with the Kolmogorov-Smirnov test. Further analyses were performed using a $t$-test for independent samples and a MannWhitney $U$-test. Statistical significance was set at $p \leq 0.05$. 
Table I. Socio-demographic profile and hip side.

\begin{tabular}{lccc}
\hline & \multicolumn{2}{c}{ Surgical technique } & \\
\cline { 2 - 3 } & With table & Without table & Total \\
\hline Patients & 97 & 92 & 189 \\
Age in years & $64(12.03)$ & $65(13.88)$ & \\
$\quad$ (Mean/range) & & & \\
Gender & 46 & 45 & 91 \\
$\quad$ Male & 51 & 47 & 98 \\
$\quad$ Female & & & \\
Hip side & 52 & 44 & 96 \\
$\quad$ Right & 45 & 48 & 93 \\
$\quad$ Left & & & \\
\hline
\end{tabular}

Values are given as mean and standard deviation.

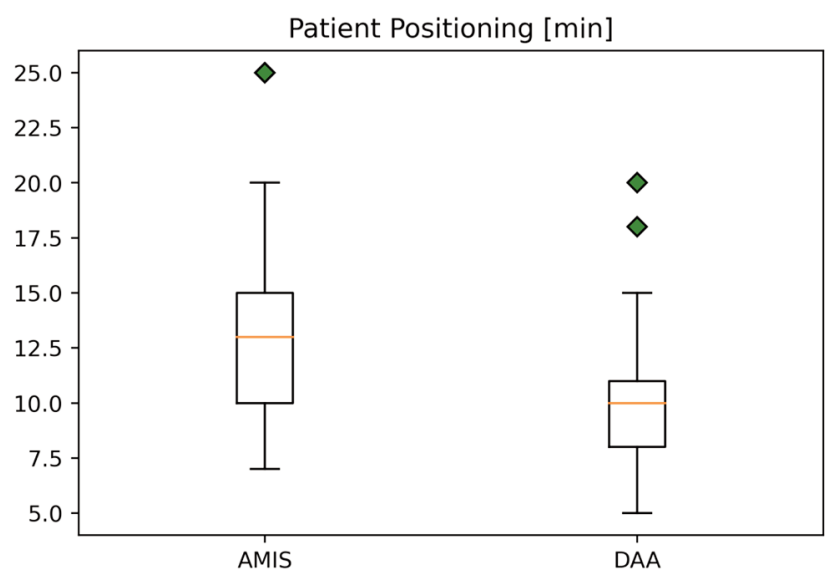

Figure 2. Time for patient positioning (time from end of anaesthesia preparation to final positioning in minutes) for AMIS ${ }^{\circledR}$ technique and conventional direct anterior approach.

\section{Results}

A total of 189 patients met the inclusion criteria and were available for evaluation. Ninety-seven patients $(51 \%)$ received THA with a traction table (AMIS ${ }^{\circledR}$ technique) and 92 patients $(49 \%)$ with conventional DAA. Sociodemographic profile and the side where the surgery was performed are shown in Table I. There was no statistically significant difference in age, sex, or side of the treated hip joint between the two above-mentioned groups.

Time for patient positioning was statistically significantly longer $(p \leq 0.001)$ in the traction table group (Figure 2$)$. Cutto-suture time was significantly shorter with traction table use $(p=0.001)$ (Table II, Figure 3). Concerning the restoration of the acetabular- $(p=0.31)$, the femoral- $(p=0.95)$ and the combined-offset ( $p=0.42)$, no statistically significant difference was found between our study groups (Table III).
Table II. Perioperative parameters.

\begin{tabular}{lccc}
\hline & With table & Without table & Total \\
\hline $\begin{array}{l}\text { Time for patient positioning } \\
\text { (min) }\end{array}$ & $13.17(3.43)$ & $9.90(2.94)$ & $<0.001$ \\
Surgical Time (min) & $74.69(15.35)$ & $81.36(14.72)$ & 0.001 \\
\hline
\end{tabular}

Values are given as mean and standard deviation.

Table III. Postoperative offset parameters.

\begin{tabular}{lrrr}
\hline & With table & Without table & Total \\
\hline Acetabular offset (mm) & $-2.57(5.88)$ & $-2.62(6.49)$ & 0.31 \\
Femoral offset (mm) & $0.70(3.81)$ & $0.94(3.97)$ & 0.95 \\
Combined offset (mm) & $-2.05(6.94)$ & $-1.75(7.04)$ & 0.42 \\
\hline
\end{tabular}

Values are given as mean and standard deviation.

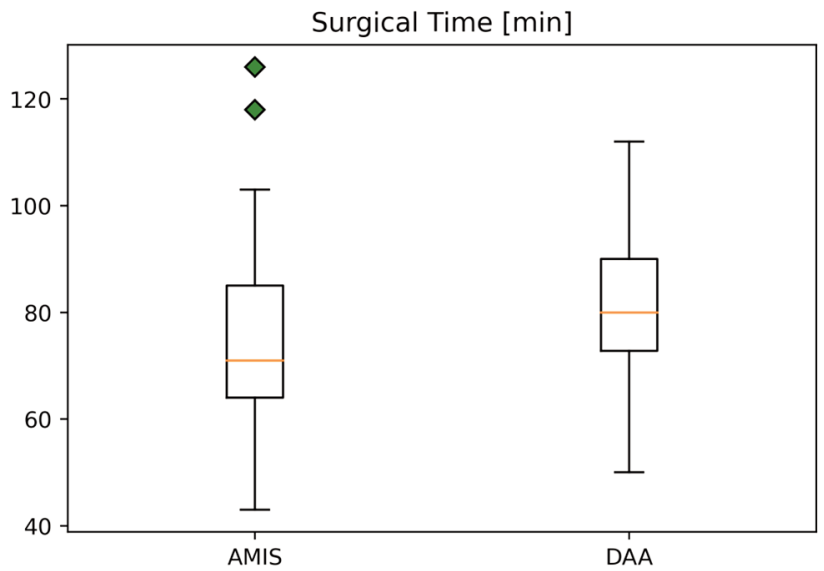

Figure 3. Surgical time (time from incision to suture in minutes) for $A M I S^{\circledR}$ technique and conventional direct anterior approach.

Postoperative leg length was statistically significantly different between the two groups $(p=0.022)$ (Table IV).

\section{Discussion}

The aim of this study was to compare operative time and accuracy of biomechanical restoration in minimally invasive THA with and without traction table use. The most important finding was the significantly shorter cut-to-suture time with the use of a traction table. Moreover, leg length restoration was significantly more accurate in the traction table group. Analysis of postoperative offset parameters revealed comparable results between the two groups. 
Table IV. Postoperative leg length discrepancy.

\begin{tabular}{lccc}
\hline & With table & Without table & Total \\
\hline $\begin{array}{l}\text { Postoperative leg length } \\
\text { discrepancy (mm) }\end{array}$ & $0.56(4.81)$ & $1.78(5)$ & 0.022 \\
\hline
\end{tabular}

Values are given as mean and standard deviation.

The shorter cut-to-suture time may be explained by the enhanced exposure, especially during femoral stem preparation, and the reliable patient position, resulting in a standardized work-flow $(18,19)$. Similar results were reported by Nakamura et al. with a mean surgical time of $72 \mathrm{~min}$ for DAA THA using a traction table (18). On the contrary, in a systematic review comparing THA via DAA with a standard and a traction table, Sarraj et al. reported a nearly $30 \mathrm{~min}$ shorter surgical duration in the standard table group. However, the analyzed studies combined positioning and surgical duration in their study and therefore, the authors explained the results with the additional time required for traction table setup (13). Wernly et al. found no significant difference when comparing duration of skin incision to wound dressing between the two methods (20). In our cohort, time for patient positioning was significantly longer in the traction table group. However, we consider reduction in surgical time as one of the key factors in preventing perioperative complications (e.g., periprosthetic infections), which outweighs the higher expenditure of time for patient positioning (21).

Reconstruction of hip biomechanics during THA is crucial for functional recovery and good clinical results (22). Inadequate offset restoration is associated with instability, diminished range of motion, edge loading, and premature polyethylene wear (23-25). In this context, Mahmood et al. demonstrated inferior clinical results in patients with a reduced combined offset of more than $5 \mathrm{~mm}$ (26). Sariali et al. published similar results. In their study, a mean decrease in femoral offset of $7.6 \mathrm{~mm}$ was associated with an increased frequency of gait disorders (27). In our results, there was no statistically significant difference concerning offset reconstruction with a slight decreased combined offset (mean $2.05 \mathrm{~mm}$ ) with traction table use (Figure 4). To our knowledge, this is the first study directly comparing postoperative offset parameters with and without traction table THA via DAA.

Substantial residual leg length discrepancy (LLD) after THA is associated with patient dissatisfaction, functional complications, and is one of the leading causes of litigation after joint replacement in the U.S. (28-30). Acceptable leg length discrepancy is still controversially discussed and clear boundaries between acceptable and unacceptable have yet to be defined. However, several studies defined a postoperative difference of $10 \mathrm{~mm}$ as acceptable, whereas other authors

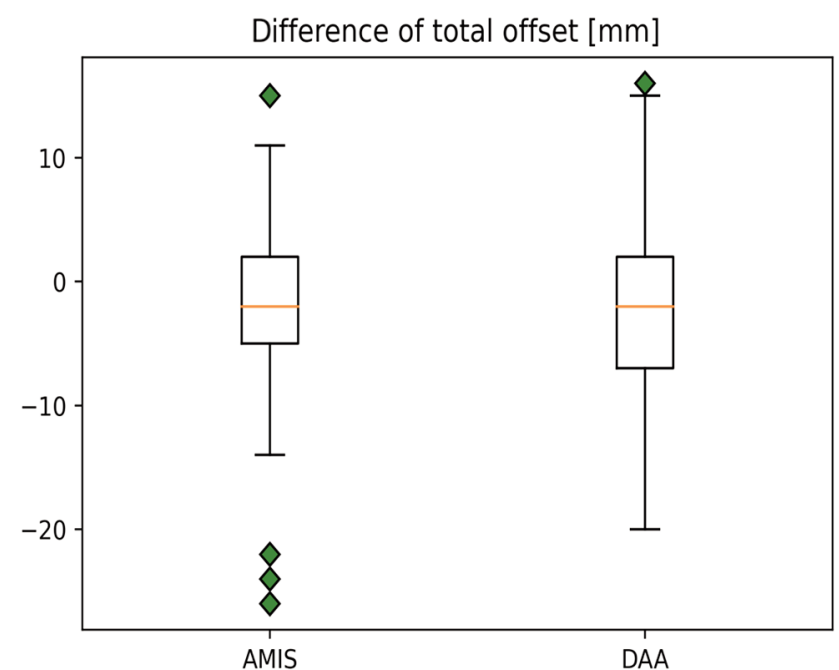

Figure 4. Difference in total offset (summarization of acetabular and femoral offset in millimeters) for $A M I S^{\circledR}$ technique and conventional direct anterior approach.

define a difference of more than $6 \mathrm{~mm}$ lengthening as pathological (31-33). THA with a standard table allows the surgeon to validate the intraoperative leg length based on anatomical land marks (e.g., medial malleoli). However, when using a traction table, the pelvis is stabilized by the perineal support, which enables standardized intraoperative fluoroscopic control and subsequently detection of leg length discrepancies. In our study, leg length reconstruction was statistically significantly more accurate in the traction table group. In contrast to our results, Wernly et al. recently reported a statistically significant more accurate leg length restoration in DAA THA without a traction table $(2.4 \mathrm{~mm} v s .3 .7 \mathrm{~mm})$. However, there was no significant difference concerning functional outcome (Harris Hip Score) (20). In a comparative study of traction table vs. standard table using DAA for THA, Moslemi et al. found no statistically significant difference concerning postoperative leg length (34).

Looking at our results with a mean difference of approximately $1.2 \mathrm{~mm}$ in postoperative leg length between the two study arms, it remains debatable if these results might have an impact on the clinical outcome. Furthermore, these results present the radiographic LLD and do not reflect the perceived leg length exactly.

This study has the following limitations: Radiological outcome was analyzed using plain radiographs instead of multidimensional imaging like computed tomography scans, EOS (EOS ${ }^{\mathrm{TM}}$ imaging) or EBRA (Einzel-BildRoentgen-Analyse). The use of such devices might have increased accuracy and comparability of our findings. Furthermore, we did not analyze the functional outcome or 
postoperative complication rate in our cohort and that somewhat limits the significance of the present results. However, this was not the major aim of our study. A further limitation is the retrospective study design and the relatively modest sample size.

In conclusion, our study presents similar radiological outcome for minimally invasive THA with and without the use of a traction table. Both methods enable restoration of hip biomechanics with high accuracy. In our opinion, the potential for shorter surgical procedure outweighs the effort and time for preparing the traction table setting. However, further studies with prospective study designs and lager sample sizes may be needed to confirm these results.

\section{Conflicts of Interest}

The Authors declare there were no conflicts of interest in regard to this study.

\section{Authors' Contributions}

$\mathrm{NH}$ was responsible for ethics approval and had the idea of the study. NH, FL, FP, FH, IL, DD, RER were involved in the initial study idea, data interpretation and article preparation. LF conducted the data collection, follow-up visits and preliminary data analysis. FP and FL conducted final data analysis and interpretation and were responsible for the final article. All Authors have read and approved the final version of the article.

\section{References}

1 Callaghan JJ, Albright JC, Goetz DD, Olejniczak JP and Johnston RC: Charnley total hip arthroplasty with cement. Minimum twenty-five-year follow-up. J Bone Joint Surg Am 82(4): 487-497, 2000. PMID: 10761939. DOI: 10.2106/ 00004623-200004000-00004

2 Sloan M, Premkumar A and Sheth NP: Projected volume of primary total joint arthroplasty in the U.S., 2014 to 2030. J Bone Joint Surg Am 100(17): 1455-1460, 2018. PMID: 30180053. DOI: $10.2106 / J B J S .17 .01617$

3 Berger RA, Jacobs JJ, Meneghini RM, Della Valle C, Paprosky W and Rosenberg AG: Rapid rehabilitation and recovery with minimally invasive total hip arthroplasty. Clin Orthop Relat Res (429): 239-247, 2004. PMID: 15577494. DOI: 10.1097/ 01.blo.0000150127.80647.80

4 Chimento GF, Pavone V, Sharrock N, Kahn B, Cahill J and Sculco TP: Minimally invasive total hip arthroplasty: a prospective randomized study. J Arthroplasty 20(2): 139-144, 2005. PMID: 15902851. DOI: 10.1016/j.arth.2004.09.061

5 Li N, Deng Y and Chen L: Comparison of complications in single-incision minimally invasive THA and conventional THA Orthopedics 35(8): e1152-e1158, 2012. PMID: 22868598. DOI: 10.3928/01477447-20120725-12

6 Tsukada S and Wakui M: Lower dislocation rate following total hip arthroplasty via direct anterior approach than via posterior approach: five-year-average follow-up results. Open Orthop J 9: 157-162, 2015. PMID: 26157532. DOI: 10.2174/1874325001 509010157
7 Tudor A, Ruzic L, Vuckovic M, Prpic T, Rakovac I, Madjarevic T, Legovic D, Santic V, Mihelic R and Sestan B: Functional recovery after muscle sparing total hip arthroplasty in comparison to classic lateral approach - A three years follow-up study. J Orthop Sci 21(2): 184-190, 2016. PMID: 26775058. DOI: $10.1016 /$ j.jos.2015.12.010

8 Imamura $\mathrm{M}$, Munro NA, Zhu S, Glazener C, Fraser C, Hutchison $\mathrm{J}$ and Vale L: Single mini-incision total hip replacement for the management of arthritic disease of the hip: a systematic review and meta-analysis of randomized controlled trials. J Bone Joint Surg Am 94(20): 1897-1905, 2012. PMID: 23079882. DOI: $10.2106 / J B J S . K .00495$

9 Stevenson C, Ogonda L, Blaney J, Dennison J, O'Brien S and Beverland D: Minimal incision total hip arthroplasty: a concise follow-up report on functional and radiographic outcomes at 10 years. J Bone Joint Surg Am 99(20): 1715-1720, 2017. PMID: 29040125. DOI: $10.2106 / J B J S .16 .00950$

10 Siguier T, Siguier M and Brumpt B: Mini-incision anterior approach does not increase dislocation rate: a study of 1037 total hip replacements. Clin Orthop Relat Res (426): 164-173, 2004. PMID: 15346069. DOI: 10.1097/01.blo.0000136651.21191.9f

11 Müller DA, Zingg PO and Dora C: Anterior minimally invasive approach for total hip replacement: five-year survivorship and learning curve. Hip Int 24(3): 277-283, 2014. PMID: 24500832. DOI: $10.5301 /$ hipint .5000108

12 Nam D, Sculco PK, Abdel MP, Alexiades MM, Figgie MP and Mayman DJ: Leg-length inequalities following THA based on surgical technique. Orthopedics 36(4): e395-e400, 2013. PMID: 23590775. DOI: 10.3928/01477447-20130327-11

13 Sarraj M, Chen A, Ekhtiari S and Rubinger L: Traction table versus standard table total hip arthroplasty through the direct anterior approach: a systematic review. Hip Int 30(6): 662-672, 2020. PMID: 31994425. DOI: 10.1177/1120700019900987

14 Dammerer D, Keiler A, Herrnegger S, Putzer D, Strasser S and Liebensteiner M: Accuracy of digital templating of uncemented total hip arthroplasty at a certified arthroplasty center: a retrospective comparative study. Arch Orthop Trauma Surg, 2021. PMID: 33725193. DOI: 10.1007/s00402-021-03836-w

15 Williamson JA and Reckling FW: Limb length discrepancy and related problems following total hip joint replacement. Clin Orthop Relat Res (134): 135-138, 1978. PMID: 729230

16 Bourne RB and Rorabeck CH: Soft tissue balancing: the hip. J Arthroplasty 17(4 Suppl 1): 17-22, 2002. PMID: 12068397. DOI: 10.1054 /arth.2002.33263

17 Flecher X, Ollivier M and Argenson JN: Lower limb length and offset in total hip arthroplasty. Orthop Traumatol Surg Res 102(1 Suppl): S9-20, 2016. PMID: 26797005. DOI: 10.1016/j.otsr. 2015.11.001

18 Matta JM, Shahrdar C and Ferguson T: Single-incision anterior approach for total hip arthroplasty on an orthopaedic table. Clin Orthop Relat Res 441: 115-124, 2005. PMID: 16330993. DOI: 10.1097/01.blo.0000194309.70518.cb

19 Nakamura J, Hagiwara S, Orita S, Akagi R, Suzuki T, Suzuki M, Takahashi K and Ohtori S: Direct anterior approach for total hip arthroplasty with a novel mobile traction table -a prospective cohort study. BMC Musculoskelet Disord 18(1): 49, 2017. PMID: 28137262. DOI: 10.1186/s12891-017-1427-2

20 Wernly D, Wegrzyn J, Lallemand G, Mahlouly J, Tissot C and Antoniadis A: Total hip arthroplasty through the direct anterior approach with and without the use of a traction table: a matched- 
control, retrospective, single-surgeon study. J Orthop Surg Res 16(1): 45, 2021. PMID: 33430917. DOI: 10.1186/s13018-02002184-6

21 Kong L, Cao J, Zhang Y, Ding W and Shen Y: Risk factors for periprosthetic joint infection following primary total hip or knee arthroplasty: a meta-analysis. Int Wound J 14(3): 529-536, 2017. PMID: 27397553. DOI: 10.1111/iwj.12640

22 Dastane M, Dorr LD, Tarwala R and Wan Z: Hip offset in total hip arthroplasty: quantitative measurement with navigation. Clin Orthop Relat Res 469(2): 429-436, 2011. PMID: 20844997. DOI: $10.1007 / \mathrm{s} 11999-010-1554-7$

23 Sakalkale DP, Sharkey PF, Eng K, Hozack WJ and Rothman RH: Effect of femoral component offset on polyethylene wear in total hip arthroplasty. Clin Orthop Relat Res (388): 125-134, 2001. PMID: 11451111. DOI: 10.1097/00003086-20010700000019

24 Sariali E, Stewart T, Jin Z and Fisher J: Three-dimensional modeling of in vitro hip kinematics under micro-separation regime for ceramic on ceramic total hip prosthesis: an analysis of vibration and noise. J Biomech 43(2): 326-333, 2010. PMID: 19892358. DOI: 10.1016/j.jbiomech.2009.08.031

25 Spalding TJ: Effect of femoral offset on motion and abductor muscle strength after total hip arthroplasty. J Bone Joint Surg Br 78(6): 997-998, 1996. PMID: 8951026.

26 Mahmood SS, Mukka SS, Crnalic S, Wretenberg P and SayedNoor AS: Association between changes in global femoral offset after total hip arthroplasty and function, quality of life, and abductor muscle strength. A prospective cohort study of 222 patients. Acta Orthop 87(1): 36-41, 2016. PMID: 26471772. DOI: $10.3109 / 17453674.2015 .1091955$

27 Sariali E, Klouche S, Mouttet A and Pascal-Moussellard H: The effect of femoral offset modification on gait after total hip arthroplasty. Acta Orthop 85(2): 123-127, 2014. PMID: 24564749. DOI: $10.3109 / 17453674.2014 .889980$

28 Desai AS, Dramis A and Board TN: Leg length discrepancy after total hip arthroplasty: a review of literature. Curr Rev Musculoskelet Med 6(4): 336-341, 2013. PMID: 23900834. DOI: $10.1007 / \mathrm{s} 12178-013-9180-0$
29 Konyves A and Bannister GC: The importance of leg length discrepancy after total hip arthroplasty. J Bone Joint Surg Br 87(2): 155-157, 2005. PMID: 15736733. DOI: 10.1302/0301620x.87b2.14878

30 Whitehouse MR, Stefanovich-Lawbuary NS, Brunton LR and Blom AW: The impact of leg length discrepancy on patient satisfaction and functional outcome following total hip arthroplasty. J Arthroplasty 28(8): 1408-1414, 2013. PMID: 23507069. DOI: 10.1016/j.arth.2012.12.009

31 Edeen J, Sharkey PF and Alexander AH: Clinical significance of leg-length inequality after total hip arthroplasty. Am J Orthop (Belle Mead NJ) 24(4): 347-351, 1995. PMID: 7788314.

32 McWilliams AB, Grainger AJ, O'Connor PJ, Redmond AC, Stewart TD and Stone MH: A review of symptomatic leg length inequality following total hip arthroplasty. Hip Int 23(1): 6-14, 2013. PMID: 23397200. DOI: 10.5301/HIP.2013.10631

33 Waibel FWA, Berndt K, Jentzsch T, Farei-Campagna J, Rahm S, Dora $\mathrm{C}$ and Zingg PO: Symptomatic leg length discrepancy after total hip arthroplasty is associated with new onset of lower back pain. Orthop Traumatol Surg Res 107(1): 102761, 2021. PMID: 33316448. DOI: 10.1016/j.otsr.2020.102761

34 Moslemi A, Kierszbaum E, Descamps J, Sigonney F, Biau D, Anract $\mathrm{P}$ and Hardy A: Does using the direct anterior approach with a standard table for total hip arthroplasty reduce leg length discrepancies? Comparative study of traction table versus standard table. Orthop Traumatol Surg Res 107(1): 102752, 2021. PMID: 33316445. DOI: 10.1016/j.otsr.2020.102752
Received November 10, 2021

Revised November 27, 2021

Accepted November 30, 2021 